\title{
Minimizing Interference in Mobile Communications Using Genetic Algorithms
}

\author{
Sa Li, Sokwei Cindy Lay, Wen Hsin Yu, and Lipo Wang ${ }^{1}$ \\ School of Electrical and Electronic Engineering, Nanyang Technological University, \\ Block S2, 50 Nanyang Avenue, Singapore 639798 \\ elpwang@ntu.edu.sg \\ ${ }^{1}$ Corresponding author
}

\begin{abstract}
There is a continuously growing demand for mobile communication. With the limited frequency spectrum, the problem of channel assignment becomes increasingly important. This problem is known to belong to a class of very difficult combinatorial optimization problems. In this paper, we apply the formulation of Ngo and Li with genetic algorithms to ten benchmarking problems, for some of which interference-free solutions cannot be found but the approach is able to minimize the interference effectively.
\end{abstract}

\section{Introduction}

In recent years, there is a continuously growing demand for mobile communication. The rate of increase in the popularity of mobile usage has very much outpaced the availability of the usable frequencies which are necessary for the communication between mobile users and the base stations of cellular radio networks. This restriction constitutes an important bottleneck for the capacity of mobile cellular systems. Careful design of a network is necessary to provide acceptable quality of service.

An important issue on the design of a cellular radio network is to determine a spectrum-efficient and conflict-free allocation of channels among the cells while satisfying both the traffic demand and the electromagnetic compatibility (EMC) constraints. This is usually referred to as a channel assignment or frequency assignment problem (CAP1). There are basically three sources of constraints [5][21], namely, co-channel constraint (CCC), where the same channel cannot be assigned to certain pairs of radio cells simultaneously; adjacent channel constraint (ACC), where channels adjacent in the frequency spectrum cannot be assigned to adjacent radio cells simultaneously; and co-site constraint (CSC), where channels assigned in the same radio cell must have a minimal separation in frequency between each other.

This channel assignment problem is equivalent to a graph-coloring problem and is thus NP-hard. Over the recent years, several heuristic approaches have been used to solve various channel assignment problems, including simulated annealing [18], neural networks [6][7][16], tabu search, and genetic algorithms 
(GAs) [11][14][19][20][22]. The existing approximative algorithm can be divided into two main groups. One group first determines ordered lists of all calls in the whole system [17] and then assign frequencies to the calls following a deterministic assignment strategy. In particular, Ngo and Li [20] developed an effective GA-base approach that obtains interference-free channel assignment by minimizing interference in a mobile network. They demonstrated that their approach efficiently converges to conflict-free solutions in some benchmarking problems.

As demand for mobile communications grows further, interference-free channel assignments often do not exist for a given set of available frequecies. Minimizing interference while satisfying demand within a given frequency spectrum is another type of channel assignment problem (CAP2) [7].

In this paper, we apply Ngo and Li's approach to several benchmarking channel assignment problems where interference-free solutions do not exist. The organization of this paper is as follows. section 2 states the channel assignment problem (CAP). In section 3 summarizes Ngo and Li's approach to solving CAP with genetic algorithms. section 4 describes the tests carried out and results obtained. Finally, section 5 concludes the paper.

\section{Channel Assignment Problem}

The channel assignment problem arises in cellular telephone networks where discrete frequency ranges within the available radio frequency spectrum, called channels, need to be allocated to different geographical regions in order to (CAP1) minimize the total frequency span, subject to demand and interferencefree constraints, or to (CAP2) minimize the overall interference, subject to demand constraints. In this paper, we are interested in CAP2.

There are two kinds of channel allocation schemes - fixed channel allocation (FCA) and dynamic channel allocation (DCA). In FCA the channels are permanently allocated to each cell, while in DCA the channels are allocated dynamically upon request. DCA is desirable, but under heavy traffic load conditions, FCA outperforms most known DCA schemes. Since heavy traffic conditions are expected in future generations of cellular networks, efficient FCA schemes is becoming more and more important [20]. The cellular network is assumed to consist of $N$ arbitrary cells and the number of channels available is given by $M$. The number of channels required (expected traffic) for cell $j$ is $D_{j}$. Assuming that the RF propagation and the spatial density of the expected traffic have already been calculated, the non-interference constraints can be determined. The electromagnetic compatibility (EMC) constraints, specified by the minimum distance by which two channels $i$ and $j$ must be separated in order to guarantee an acceptably large signal-to-interference ratio $S / I$ within the regions to which the channels are assigned, can be represented by an $N \times N$ symmetric matrix called the compatibility matrix $C=\left\{C_{i j}\right\}$.

The solution space is represented by $F$ as an $N \times M$ binary matrix, where $N$ is the total number of radio cells and $M$ is the total number of available channels 
[20]. Each element $f_{j k}$ in the matrix is either one or zero such that

$$
f_{j k}=\left\{\begin{array}{l}
1, \text { if channel } \mathrm{k} \text { is assigned to cell } j ; \\
0, \text { otherwise }
\end{array}\right.
$$

The cellular network is expected to meet the demand of the traffic and to minimize all forms of interference. The first requirement is the demand constraint, i.e., for cell $i$, a total of $D_{i}$ channels are required. This implies that the total number of ones in row $i$ of $F$ must be $D_{i}$. If the assignment to cell $i$ violates the demand constraint, then

$$
\left(\sum_{q=1}^{M} f_{j q}-D_{i}\right) \neq 0 \text {. }
$$

The second requirement depends on the compatibility matrix $C$. It is made up of CSC, CCC and ACC. In order to satisfy the CSC, if channel $p$ is within distance $C_{i i}$ from an already assigned channel $q$ in cell $i$, then channel $p$ must not be assigned to cell $i$. This can be seen from the equation below:

$$
\sum_{q=p-\left(C_{i i}-1\right), q \neq p, 1 \leq q \leq m}^{p+\left(C_{i i}-1\right)} f_{i q}>0 .
$$

To satisfy the requirements for CCC and ACC, if channel $p$ in cell $i$ is within distance $C_{i j}$ from an already assigned channel $q$ in cell $j$, where $C_{i j}>0$ and $i \neq j$, then channel $p$ must not be assigned to cell $i$. This is represented as follows:

$$
\sum_{j=1, j \neq i, C_{i j}>0}^{N} f_{j q} \sum_{q=p-\left(C_{i i}-1\right), q \neq p, 1 \leq q \leq m}^{p+\left(C_{i i}-1\right)} f_{i q}>0 .
$$

Therefore, the cost function of CAP can be expressed as

$$
\begin{array}{r}
C(F)=\sum_{i=1}^{N} \sum_{p=1}^{M}\left(\sum_{j=1, j \neq i, C_{i j}>0}^{N} \sum_{q=p-\left(C_{i i}-1\right), q \neq p, 1 \leq q \leq m}^{p+\left(C_{i i}-1\right)} f_{i q}\right) f_{i p} \\
+\alpha \sum_{i=1}^{N} \sum_{p=1}^{M}\left(\sum_{q=p-\left(C_{i i}-1\right), q \neq p, 1 \leq q \leq m}^{p+\left(C_{i i}-1\right)} f_{i q}\right) f_{i p}+\beta \sum_{i=1}^{N}\left(\sum_{q=1}^{M} f_{j q}-D_{i}\right),
\end{array}
$$

where $\alpha$ and $\beta$ are weighting factors. It is noted that $C(F)$ achieves its minimum of zero when all constraints are satisfied. So, the objective of our problem is to find an $F$ such that $C(F)$ is minimized.

\section{Solving Channel Assignment Problem with Genetic Algorithm by Ngo and Li}

In the encoding scheme by Ngo and Li [20], a $p$-bit binary string represents an individual with $q$ fixed elements and the minimum separation between consecutive elements is represented by $d_{\min }$. The concept of this scheme is to represent 
the solution in a way such that a one is followed by $d_{\min }-1$ zeros encoded as a new "one", denoted as I. Using the minimum separation scheme, the cost function can be simplified by exploiting the symmetry of the compatibility matrix $C$. Hence, the final cost function is represented by

$$
\begin{gathered}
C(F)=\sum_{i=1}^{N-1} \sum_{j=i+1, C_{i j}>0}^{N}\left(\sum_{p=1}^{C_{i j}-1} \sum_{q=1}^{p-1} f_{j q} f_{i p}\right. \\
\left.+\sum_{p=C_{i j}}^{M} \sum_{q=p-C_{i j}+1}^{p-1} f_{j q} f_{i p}+\frac{1}{2} \sum_{p=1}^{M} f_{j q} f_{i p}\right) .
\end{gathered}
$$

The mechanism of genetic algorithms start with randomly generating a population of chromosomes (suitable solutions for the population). The solution space represented by $F$, a $N \times M$ matrix, is treated as a chromosome in the population. This means that if a population is to contain $\mathrm{n}$ chromosomes, there will be $\mathrm{n}$ $F$ solution matrixes in the population, each representing a chromosome. These n $F$ solution matrixes or arrays are randomly generated and are all possible solutions for the channel assignment problem. The number of chromosomes in a population is stated by the population size, which is a parameter that should be manipulated to obtain an optimized solution. The setting of population size is generally quite ad hoc but nevertheless, a relatively small population size is suggested [20].

After randomly generating a population of chromosomes, the fitness of each chromosome should be evaluated. Therefore, all $F$ solution arrays in the population are evaluated for their fitness values [20][20][20], by using the final cost function eq.(5). The lower the cost function value, the fitter the chromosome.

The next step in genetic algorithm is to generate a new population, using genetic algorithm operators, such as selection, crossover, and mutation. The selection process consists of selecting 2 parent chromosomes from a population according to their fitness (the better fitness, the bigger chance to be selected). The selected chromosomes have to undergo minimum-separation encoding, to ensure that the CSC satisfaction in them will continue to be satisfied even after crossover and mutation.

After selection and encoding, the selected parent chromosomes, or selected $F$ solution arrays (encoded), will undergo crossover, with a probability of crossover, and mutation with a probability of mutation. Crossover probability and mutation probability are parameters that should be manipulated to obtain an optimized solution. The settings of these parameters, like the population size parameter, are generally quite ad hoc but a high crossover probability and low mutation probability are suggested [20]. Therefore, after crossover and mutation, the new offspring of the parent chromosomes are placed in the new population. For parents whereby no crossover or mutation is performed, they will be placed in the new population too. The selection, crossover and mutation processes will be repeated till the new population, which has the same size of the old population, is formed. After which, all new $F$ solution arrays, or chromosomes, will be used for a further run of the entire genetic algorithm till an optimized solution is found. 


\section{Application of the Ngo-Li GA Approach to Benchmarking CAPs}

The data sets used to test the performance of the approach were taken from [7]. Ten benchmarking problems were examined. Table 1 lists the problem specification and Table 2 lists the demands of those problems. The CPU time taken

Table 1. Problem specifications of the ten benchmarking problems used. $\left[D_{5}\right]_{i}$ stands for the first $i$ elements of matrix $\left[D_{5}\right]$.

\begin{tabular}{|c|c|c|c|}
\hline Problem & No. Cell & No. Channel & Demand \\
\hline EX1 & 4 & 11 & $D_{1}$ \\
EX2 & 5 & 17 & $D_{2}$ \\
\hline HEX1 & 21 & 37 & $D_{3}$ \\
HEX2 & 21 & 91 & $D_{3}$ \\
HEX3 & 21 & 21 & $D_{4}$ \\
HEX4 & 21 & 56 & $D_{4}$ \\
\hline KUNZ1 & 10 & 30 & {$\left[D_{5}\right]_{10}$} \\
KUNZ2 & 15 & 44 & {$\left[D_{5}\right]_{15}$} \\
KUNZ3 & 20 & 60 & {$\left[D_{5}\right]_{20}$} \\
KUNZ4 & 25 & 73 & $D_{5}$ \\
\hline
\end{tabular}

Table 2. Demand constraints of the benchmarking problems.

\begin{tabular}{|c|c|}
\hline & Demand \\
\hline$D_{1}^{T}$ & $(1,1,1,3)$ \\
$D_{2}^{T}$ & $(2,2,2,4,3)$ \\
\hline$D_{3}^{T}$ & $(2,6,2,2,2,4,4,13,19,7,4,4,7,4,9,14,7,2,2,4,2)$ \\
$D_{4}^{T}$ & $(1,1,1,2,3,6,7,6,10,10,11,5,7,6,4,4,7,5,5,5,6)$ \\
\hline$D_{5}^{T}$ & $(10,11,9,5,9,4,5,7,4,8,8,9,10,7,7,6,4,5,5,7,6,4,5,7,5)$ \\
\hline
\end{tabular}

for each problem was dependent on the number of the size of the population used. A bigger population would take a much longer time for the minimum cost function to be found. Figure 1 shows the typical rate of convergence trajectory based on CPU time. Figure 2-3 shows the typical rate of convergence trajectory based on number of generations. Figures 4-7 show the sub-optimal solutions of channel assignments obtained from GA. Each dot represents a traffic demand for a particular cell and this demand would be allocated to a channel in a manner such that all the interferences are minimized. During the simulation, several parameters, such as crossover probability, mutation probability and population size, need to be set. The number of generations for different problems also needs 


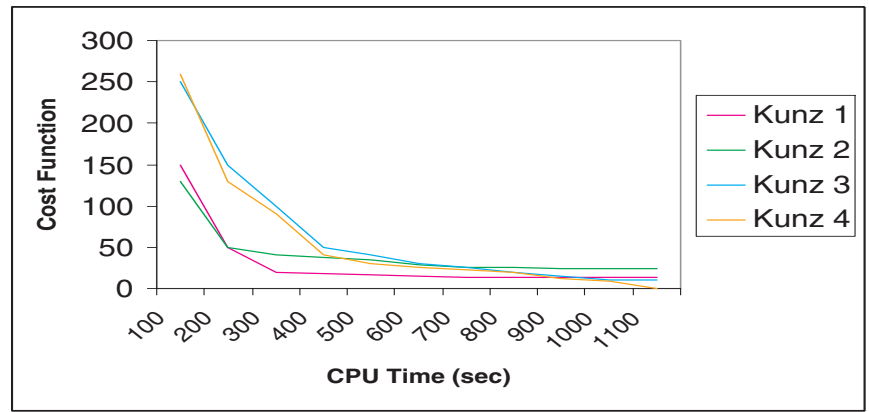

Fig. 1. A typical rate of convergence trajectory based on CPU time.

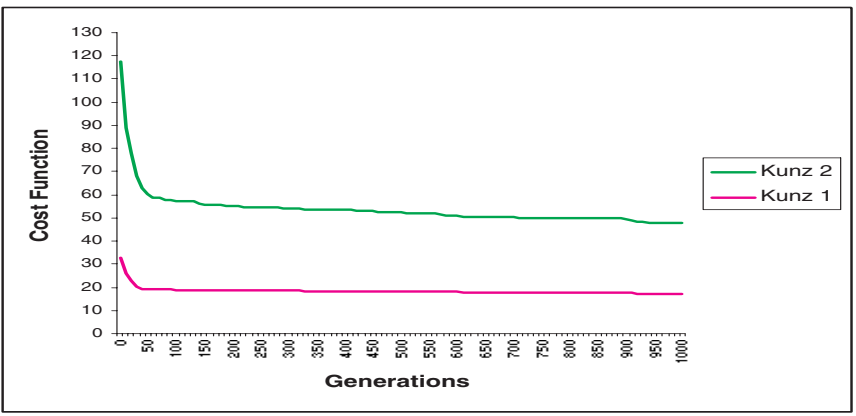

Fig. 2. A typical rate of convergence trajectory based on number of generations for KUNZ1 and KUNZ2.

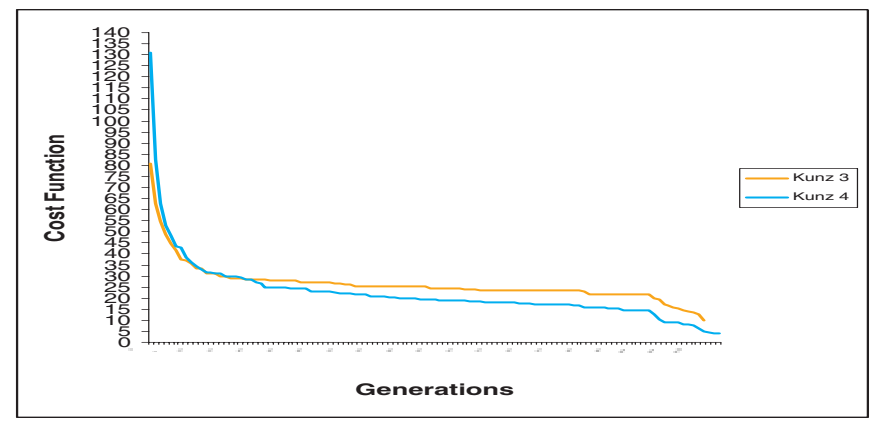

Fig. 3. A typical rate of convergence trajectory based on number of generations for KUNZ3 and KUNZ4. 


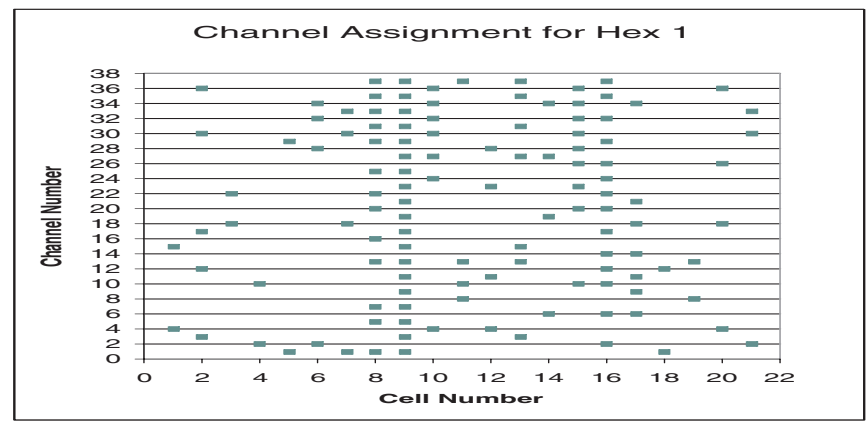

Fig. 4. Channel assignment for Hex 1 with interference value 39

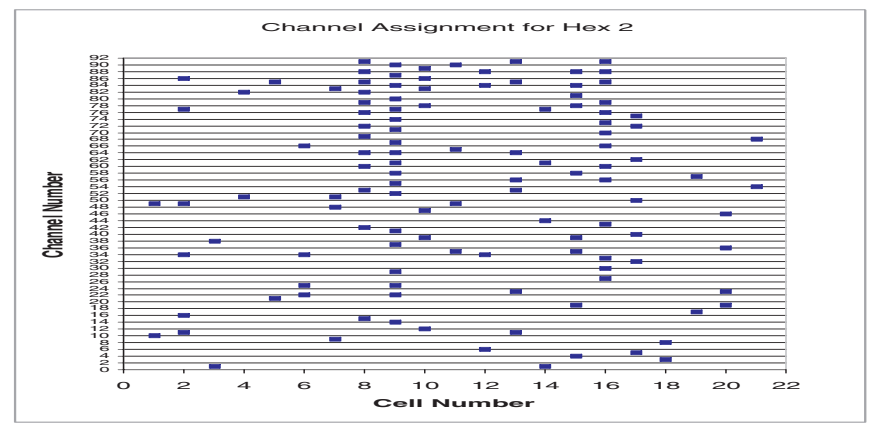

Fig. 5. Channel assignment for Hex 2 with interference value 13.5

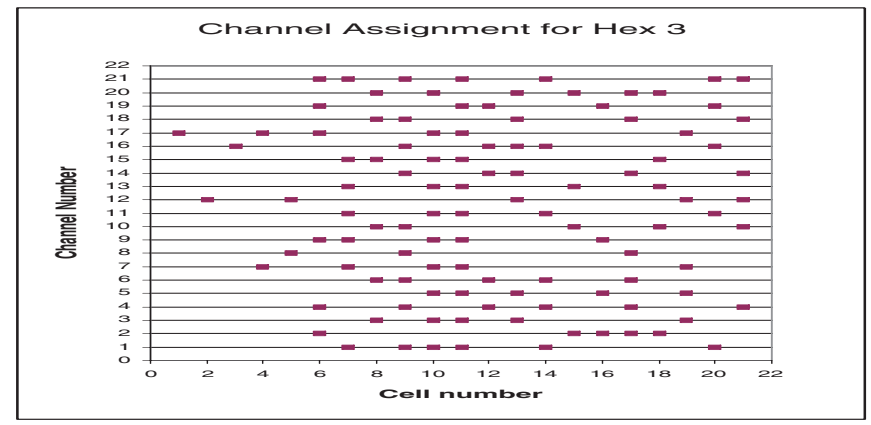

Fig. 6. Channel assignment for Hex 3 with interference value 46.5 


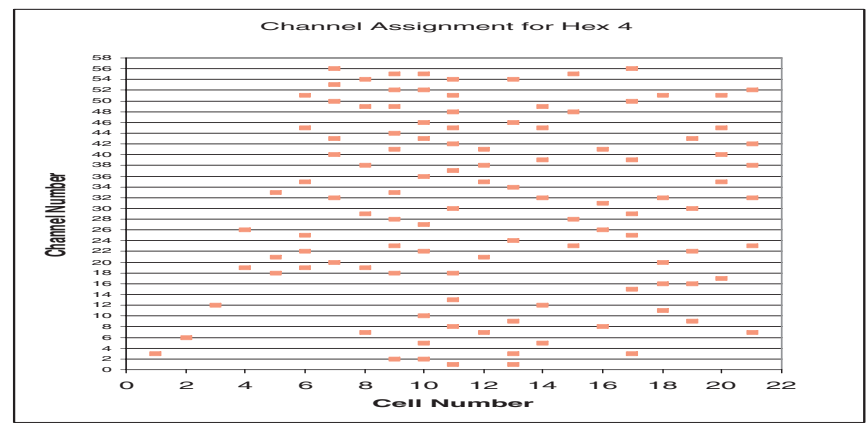

Fig. 7. Channel assignment for Hex 4 with interference value 0

to be taken into account. For example, for larger problems like Kunz 4, the number of generations needed to obtain a satisfactory result is 100000 as compared to 50000 for a smaller problem Kunz 3. For any genetic algorithms, the settings of these parameters are generally by trial-and-error. One general rule was kept throughout the simulation as suggested in [4]. That is to use relatively small population size, high crossover probability, and low mutation probability. We have systematically tested various choices of these parameters. For example, when we set the population size as 20 and the mutation probability as 0.004 , the following tables show how the cost function changes with the cross-over probability:

\section{Conclusion}

In this paper, we applied Ngo and Li's GA-based approach to CAP2, i.e., channel assignment problems in which the total interference is minimized while traffic demands are satisfied within a given set of available channels. This approach permits the satisfaction of traffic demand requirement and co-site constraint. It is achieved by the use of a minimum-separation encoding scheme, which reduces the required number of bits for representing the solution, and with unique genetic operators that kept the traffic demand in the solution intact. This allowed the search space to be greatly reduced and hence shorten the computation time. The simulations done on benchmark problems showed that this approach could achieve desirable results.

Although we have tested a variety of choices of parameters, such as mutation rate, cross-probability, and population size, more such test with other choices of parameters should be carried out. Implementations of GAs for DCA will also be studied in future work.

\section{References}

1. Michalewicz, Z., "Genetic Algorithms + Data Structures = Evolution Programs." Berlin; New York: Springer-Verlag, c1994. 
Table 3. Effect of Different Probability of Crossover for Hex

\begin{tabular}{|c|c|c|}
\hline Problem & Probability of Crossover & Cost Function \\
\hline HEX1 & 0.85 & 35 \\
& 0.90 & 34 \\
& 0.95 & 33 \\
& 0.97 & 33.5 \\
& 0.99 & 34 \\
\hline HEX2 & 0.85 & 14 \\
& 0.90 & 14 \\
& 0.95 & 13.5 \\
& 0.97 & 14.5 \\
& 0.99 & 14.5 \\
\hline HEX3 & 0.85 & 48.5 \\
& 0.90 & 48 \\
& 0.95 & 46.5 \\
& 0.97 & 47 \\
& 0.99 & 47.5 \\
\hline HEX4 & 0.85 & 16 \\
& 0.90 & 15 \\
& 0.95 & 14.5 \\
& 0.97 & 15.5 \\
\hline
\end{tabular}

2. Lai, W. K., Coghill, G. G. , "Channel assignment through evolutionary optimization", IEEE Trans. Veh. Technol., vol. 45, no. 1, pp.91-96, 1996.

3. Ngo, C. Y., "Genetic algorithms for discrete optimization and their applications to radio network design", Ph.D. thesis, Dept. Electr. Eng. Syst., Univ, Southern California, Los Angeles, CA., Aug.1995.

4. Goldberg, D. (Edward, D.), "1953- genetic algorithms in search, optimization, and machine learning." Reading, Mass.: Addison-Wesley Pub. Co., 1989.

5. Sivarajan, K. N., McEliece, R. J., "Channel Assignment in Cellular Radio", Proceedings IEEE Vehicular Technology Conference, vol. 68, pp. 1497-1514, Dec., 1980.

6. Kunz, D., "Channel assignment for cellular radio using neural networks", IEEE Trans. Veh. Technol., vol. 40, pp. 188-193, Feb 1991

7. Smith, K., Palaniswami, M., "Static and dynamic channel assignment using neural network", IEEE Journal on Selected Areas in Communications, vol. 15, no. 2, Feb., 1997.

8. Holland, J. H., "Adaptation in Natural and Artificial Systems", Ann Arbor, MI: Univ. Michigan Press, 1975.

9. Falkenauer, E., "Genetic algorithms and grouping problems", Chichester, England: Wiley, 1998

10. Davis, L., "Genetic Algorithms and Simulated Annealing", London, U.K.: Pitman, 1987.

11. Lai, W. K., Coghill, G. G., "Channel Assignment for a Homogeneous Celular Network with Genetic Algorithms", Conf. Intelligent Information Systems, pp. 263267, 1994. 
12. Marin, J., Sole, R. V., "Macroevolutionary Algorithms: A New Optimization Method on Fitness Landscapes", IEEE Transations on Evolutionary Computation, vol. 3, no. 4, 1999.

13. Hata, M., "Empirical Formula for Propagation Loss in Land Mobile Radio Service", IEEE Trans. Veh. Technol., vol.29, no.3, pp.317-325, 1980.

14. Cuppini, M., "A genetic algorithm for channel assignment problems", Eur. Trans. Telecomm. Related Technol., vol.45, no.1, pp.91-96, 1996.

15. Kim, S., Kim, S. L., "A two-phase algorithm for frequency assignment in cellular mobile systems", IEEE Trans. Veh. Technol., vol. 43, no. 3, pp. 542-548, 1994.

16. Funabiki, N., Takefuji, Y., "A neural network parallel algorithm for channel assignment problems in cellular radio networks", IEEE Trans. Veh. Technol., vol. 41, no. 4, pp. 430-437, 1992.

17. Ko, T. M., "A frequency selective insertion strategy for fixed channel assignment", Proc. 5th IEEE int, Symp.Personal, Indoor and Mobile Radio Commun, pp. 311314, Sept. 1994.

18. Mathar, R., Mattfeldt, J., "Channel assignment in cellular radio networks", IEEE Trans. Veh. Technol., vol. 42, pp. 14-21, 1993.

19. Kim, J. S., Park, S. H., Dowd, P. W., Nasrabadi, N. M., "Channel assignment in cellular radio using genetic algorithm", Wireless Personal Commun. vol. 3, no. 3, pp. 273-286, Aug. 1996.

20. Ngo, Y., Li, V. O. K., "Fixed channel assignment in cellular radio networks using a modified genetic algorithm", IEEE Trans. Veh. Technol., vol. 47, no. 1, 1998.

21. Ko, T. M., "A frequency selective insertion strategy for fixed channel assignment", Proc. 5th IEEE Int. Symp. Personal, Indoor and Mobile Radio Commun., pp. 311314, 1994.

22. Smith, K., "A genetic algorithm for the channel assignment problem", IEEE Global Technology Conference., vol. 4, 1998. 